Archives

17 | 1996

Hommage à Bernard Lepetit

\title{
Géologique sous le second empire. L'expédition scientifique du Mexique (1864-1867)
}

\section{Claire Fredj}

\section{Q OpenEdition}

Journals

Édition électronique

URL : http://journals.openedition.org/ccrh/2630

DOI : $10.4000 /$ ccrh.2630

ISSN : $1760-7906$

Éditeur

Centre de recherches historiques - EHESS

Édition imprimée

Date de publication : 4 octobre 1996

ISSN : 0990-9141

Référence électronique

Claire Fredj, « Géologique sous le second empire. L'expédition scientifique du Mexique (1864-1867) », Les Cahiers du Centre de Recherches Historiques [En ligne], 17| 1996, mis en ligne le 27 février 2009, consulté le 01 mai 2019. URL : http://journals.openedition.org/ccrh/2630 ; DOI : 10.4000/ccrh.2630

Ce document a été généré automatiquement le 1 mai 2019.

Article L.111-1 du Code de la propriété intellectuelle. 


\title{
Géologique sous le second empire. L'expédition scientifique du Mexique (1864-1867)
}

\author{
Claire Fredj
}

1 En février 1864, deux ans après les débuts de la campagne militaire, Napoléon III et Victor Duruy organisent, sur le modèle explicite de l'expédition d'Égypte, une expédition scientifique au Mexique. Une commission scientifique, basée au ministère de l'Instruction publique, composée d'environ 25 personnes, la plupart membres de l'Institut, dirige un réseau de correspondants, en France et au Mexique. Divisée en quatre comités spécialisés, la commission donne des instructions aux voyageurs qu'elle a désignés ${ }^{1}$.

2 Dépendant du Comité des sciences naturelles et médicales, deux de ces voyageurs, Dollfus et Montserrat, géologues, constituent l'objet de mon étude. En suivant leurs itinéraires lors de l'expédition, en analysant de manière sommaire leur production scientifique, mais aussi la manière dont se fabrique cette production, je souhaite montrer les modes d'adaptation de ces deux voyageurs à un terrain donné, les réajustements constants, leur inscription dans plusieurs espaces de pratiques, de connaissances, d'expériences permettant de saisir leur compréhension des territoires parcourus. Processus de redéfinition des contours, construction d'espaces multiples, attention aux conditions matérielles de production du savoir, analyse fine du discours destinée à « transformer un texte plat en mystère $»^{2}$, autant de pistes que Bernard Lepetit explorait, que je n'aurais pas songé un jour prospecter sans lui.

\section{Auguste Dollfus et Eugène Montserrat, voyageurs}

3 À l'annonce de la formation de la mission scientifique, le ministère reçoit des demandes spontanées de participation. Pour la géologie, deux étudiants, se présentant comme "ingénieurs civils», posent leur candidature. Nés en 1840, ces deux hommes sont recommandés par Charles Sainte-Claire-Deville ${ }^{3}$, qui s'en occupera pendant toute la durée de leur voyage. 
4 Auguste Dollfus est élève en troisième année à l'École des mines. Auteur d'un ouvrage sur La Faune kiméridienne du cap de la Hève (1863), membre de la Société de géologie, il a publié quelques-unes de ses recherches dans le bulletin de cette institution. Il fait partie de l'expédition scientifique comme "voyageur photographe et paléontologiste ». Également élève en troisième année, mais à l'École centrale des arts et manufactures, Eugène de Montserrat est nommé comme « voyageur géologue plus spécialement chargé de l'étude des formations volcaniques $»^{4}$. Pendant quelque temps, ils sont accompagnés par Paul Pavie, 27 ans, ex-étudiant en médecine.

Devant d'abord s'étendre « des sources du Rio del Norte et du Rio Colorado jusqu'au golfe de Darien, sur environ $32^{\circ}$ de latitude $\aleph^{5}$, le champ d'exploration se réduit essentiellement au Yucatan ${ }^{6}$, exception faite pour les géologues et les minéralogistes, qui vont là où il $\mathrm{y} a$ des mines. Les explorations de Dollfus et Montserrat devraient donc les conduire dans le nord du pays.

Chaque voyageur est censé collecter le plus de données possibles dans les domaines des sciences naturelles, de la météorologie, de l'ethnologie et de tout ce qui concerne le Mexique. Ensuite viennent des instructions plus précises, relatives à la spécialité de chacun.

7 En ce qui concerne la géologie, l'objectif final est la réalisation de la carte géologique du Mexique. Objectif immédiatement reconnu comme prématuré :

Il s'agit donc pour le moment de recueillir des données générales sur la constitution géognostique [du pays] ou si l'on veut d'élargir et de compléter celles qu'on doit à un petit nombre d'observateurs?

On demande aux voyageurs de

[...] fixer clairement, par des monographies... les rapports et les anomalies qu'on peut observer entre les formations éruptives et sédimentaires de l'Amérique centrale et celle de l'Europe ou de l'Amérique du Nord.

Suivent les terrains à examiner.

Pour réaliser ce programme, il faut se préparer: lectures sur la géologie mexicaine, examen de collections minéralogiques, étude sur les lieux de la

[...] géologie de quelques points peu éloignés de nos frontières qui offrent des analogies frappantes avec les formations [à examiner] au Mexique ${ }^{8}$.

C'est ainsi que Dollfus, Montserrat et Pavie partent étudier

[...] les terrains volcaniques des bords du Rhin ainsi que les formations porphyriques et granitiques du Palatinat et de l'Odenwald'.

Le choix de ces régions est dicté par le vade-mecum d'Ami Boué, largement utilisé par les géologues de son temps ${ }^{10}$. Lectures et voyage préliminaire s'intègrent parfaitement à la méthode de travail du géologue : on lit, par exemple, chez Huot qu'avant de visiter des pays peu connus, il faut

[...] visiter de préférence les contrées classiques qui, ayant été bien étudiées, présentent une foule de localités instructives ${ }^{11}$.

Penser que l'on peut étudier le connu proche européen pour comprendre l'inconnu éloigné est un des postulats de la méthode géologique, qui repose sur la constatation que

[...] la succession de tous les dépôts de sédiments et celle de roches ignées de toute la surface du globe [ont] pu être rigoureusement établies par la connaissance seule de quelques parties de l'Allemagne, de la Grande-Bretagne, de la France et de l'Italie, comme si la nature eût voulu que cette Europe occidentale qui devait être le berceau des sciences modernes pût offrir en même temps un abrégé complet de la plus vaste d'entre elles ${ }^{12}$. 
L'Europe apparait donc comme le modèle auquel on rapportera d'autres structures ${ }^{13}$, même si ce modèle ne permet pas toujours de tout comprendre ${ }^{14}$.

\section{Les difficultés de l'expédition mexicaine et sa réorientation vers l'Amérique centrale}

Dès l'arrivée,

[...] « désirant mettre immédiatement à profit notre séjour au Mexique, nous avons entrepris de faire à pied, avec un corps de troupes, le voyage de la Vera Cruz à Mexico, ce qui nous a permis de recueillir en chemin des matériaux assez nombreux » et d'envoyer ainsi plusieurs travaux géologiques relatifs à cette portion du Mexique ${ }^{15}$.

11 Basés à Mexico en attendant, nos géologues ont du mal à éviter les contraintes de la guerre, cette autre science du terrain. À cause des «troubles", le Mexique offre «un champ de travail presque nul $»^{16}$ puisque

[...] les faibles secours que nous espérions obtenir des autorités militaires et avec lesquelles nous aurions peut-être pu faire quelque chose ont dû nous être absolument refusés...

De l'armée dépend, en effet, une bonne partie des travaux des trois hommes: une excursion au sommet du Popocatepetl est ainsi possible grâce à la protection d'une escorte armée fournie par le général Bazaine. Pourtant les voyageurs n'ont pas pu faire toutes les observations qu'ils auraient voulu, d'abord à cause du mauvais temps, ensuite à cause

[des] exigences du service militaire, relatives à notre escorte, qui ne nous était accordée que pour un temps très restreint ${ }^{17}$.

L'espoir est suspendu aux mouvements de troupes :

Dans quelques jours, nous allons partir pour Guanajuato...: nous sommes contraints, pour nous y rendre, de suivre une colonne de troupe qui va rejoindre l'armée dans le Nord...

Parfois, cependant, savants et militaires peuvent coopérer de manière efficace : sont mentionnés, par exemple,

[...] des panoramas photographiques exécutés en commun par M. Pavie et le capitaine Riffault, qui a bien voulu mettre au service du missionnaire de la commission ses connaissances et son habileté pratique en photographie ${ }^{18}$.

De même, après avoir dû renoncer à une excursion dans le Michoacan " par suite de l'état du pays ", les voyageurs peuvent concentrer leurs recherches

[...] dans deux districts voisins de Sultepec et de Tasco, dont les autorités facilitent [leurs] travaux de toutes les manières ${ }^{19}$.

14 On voit ainsi comment la réalité redessine le programme élaboré à Paris. Redéfinissant leur itinéraire et les objectifs de leur mission, nos voyageurs font progresser la science par un autre chemin :

Débarqués à La Union, extrémité sud du Salvador, nous comptons étudier ce pays très intéressant au point de vue géologique, gagner ensuite le Guatemala et de là Mexico, en traversant les provinces du sud du Mexique, fort peu explorées jusqu'ici ${ }^{20}$.

15 Libérés des contraintes militaires,

[...] montés sur nos chevaux ${ }^{21}$, suivis par deux domestiques mexicains... accompagnés de deux mules portant quelques vivres, quelques effets et 
instruments scientifiques, parcourant tantôt les sentiers battus, tantôt les sentiers où ne passe guère que l'Indien voyageur, nous avons marché devant nous... examinant tout ce que la nature nous offrait de sujets d'étude ${ }^{22}$. inhospitalières... »; ils peuvent avoir du mal à trouver un guide ;

[les] habitants du pays ne songent guère, en général, à s'occuper des spéculations scientifiques ${ }^{24}$.

Leurs travaux vont pourtant se trouver considérablement facilités par leur insertion dans un réseau de connaissances et d'institutions : ils sont aidés par des personnages officiels comme les présidents des républiques du Salvador et du Guatemala, par différents ministres, par des consuls. Ils se servent également des institutions savantes locales, telle la Sociedad económica du Salvador, qui leur ouvre son dépôt scientifique et sa bibliothèque, ou encore le Collegio Tridentino de Guatemala, dont ils utilisent les collections minérales et surtout l'observatoire ${ }^{25}$. Enfin, des particuliers - comme les frères Oscar et Xavier Du Theil, Jules Rossignon (correspondants de la commission), les frères Georges et Henri Bramma - leur ouvrent leurs haciendas et les font bénéficier de leur connaissance du terrain.

Cette inscription dans un espace administratif et scientifique se répercute sur la forme de leur travail. Le résultat « savant» de leur voyage en Amérique centrale, leur livre publié par le ministère de l'Instruction publique dès 1868 , insère un grand nombre d'études et de documents provenant de ces cercles. Cet ouvrage, véritable consécration pour de jeunes savants, réutilise également d'autres observations indirectes, comme des extraits de livres sur l'Amérique centrale ${ }^{26}$, de journaux ${ }^{27}$, reliant nos savants à des espaces de connaissance plus larges.

\section{La science telle qu'elle se fait : l'évolution des choix scientifiques face à l'inconnu}

Lettres et rapports contiennent de nombreuses notations concernant les instruments utilisés.

En permanence en train de recueillir des données, nos voyageurs ont porté partout leur baromètre avec eux ${ }^{28}$.

Ils transportent aussi thermomètres, baromètres, théodolites; ils analysent les eaux des lacs avec des réactifs, recueillent des gaz dans des tubes en verre « où le vide avait été fait préalablement ${ }^{29}$. Tout cela concerne surtout le travail « généraliste» imposé par les instructions. Peu de références en revanche à leur métier de géologue (coupes, profils, cartes, croquis) ; le discours savant sur les formations du sol est le résultat d'un travail technique dont on a peu de traces.

Les Cahiers du Centre de Recherches Historiques, 17 | 1996 
À quoi peuvent bien servir les très nombreux et hétéroclites instruments que le ministère fait acheter à leur intention pour une somme de 2101,79 francs $^{30}$ ? Comment arrivent-ils à l'étude du

[...] sol lui-même, envisagé au point de vue de sa constitution intime, des variétés de roches qui le composent, des origines de son relief actuel ${ }^{31}$ ?

Mettre en lumière les contours de cet espace disciplinaire passe également par l'étude du discours de Dollfus et Montserrat sur leur travail.

Le travail du géologue consiste en l'étude lithologique proprement dite mais aussi comporte une bonne part de spéculation, ce qu'ils appellent " géologie théorique »,

[qui consiste à] trouver dans la direction des montagnes ou dans les alignements des points composés de la même roche des éléments qui puissent... aider à rechercher l'âge relatif des assises ou leur développement horizontal ${ }^{32}$.

Pour se livrer à cet exercice, il faut pallier un obstacle de taille: "l'insuffisance des données géographiques ». Seule une carte topographique exacte permettra de faire des hypothèses. Les géologues se transforment donc en topographes. Cette polyvalence est indispensable comme le montrent les ouvrages de géologie, qui insistent par ailleurs sur l'insuffisance de cette connaissance de surface, purement géographique ${ }^{33}$. Les voyageurs tracent donc leur propre carte, résultat d'une synthèse entre « toutes les déterminations précises utilisées déjà pour d'autres cartes » et les points rectifiés "d'après [leurs] travaux, tout ce qui ne... semblait pas pouvoir concorder avec [leurs] observations $»^{34}$.

Ce document se présente comme le résumé graphique de leur voyage : il marque à la fois leurs déplacements dans le pays, les renseignements collectés, leurs travaux effectifs et leurs interrogations. Tout cela se rejoint visuellement sur la carte au moyen du dispositif graphique suivant :

Nous avons d'abord reporté tous nos itinéraires géologiques, puis certains renseignements géologiques qui nous ont été communiqués, et nous avons figuré la nature $d u$ sol en teintes plates, [...] dans tous les points dont la constitution géologique nous était connue d'une façon incontestable. Les mêmes couleurs en teintes fondues ont été appliquées sur les localités où la composition du terrain ne nous était connue que par des renseignements vagues ou par des hypothèses plus ou moins plausibles; enfin nous avons laissé en blanc les régions sur lesquelles nous ne possédons aucune notion..$^{35}$.

La forme de ce bilan de terrain reconnu professionnellement répond à une hypothèse de travail précise. Le parcours à travers l'Amérique centrale s'inscrit dans un projet méthodique, défini d'après un double manque : manque de temps et manque quasi total d'informations sur le terrain du point de vue géologique, à part quelques notations éparses dans les ouvrages parus sur la région ${ }^{36}$.

Deux possibilités s'offrent alors : soit s'intéresser à quelques points saillants du territoire et produire des monographies " complètes, sérieuses, d'une véracité indiscutable ", qui, reliées entre elles, donneront une connaissance complète du pays; soit procéder au «tracé de la gigantesque esquisse posée par les soins de la nature,... quitte à ne pas toujours approfondir $\|^{37}$. Chaque méthode a ses défauts. Pour la première, c'est le risque de confusion qui peut naître de la juxtaposition de monographies élaborées par différents auteurs, donc à chaque fois « reflet d'opinion personnelle $»^{38}$. Pour la deuxième, le risque d'erreur est lié à une trop grande généralisation.

En fait, l'apparente neutre des hypothèses de travail justifie un choix, en disqualifiant une méthode au profit d'une certaine conception de l'aventure. Lorsque les auteurs écrivent : 
Nous eussions pu nous borner à l'examen de quelques-uns des magnifiques

volcans... recueillir ainsi les matériaux de quelques mémoires savamment étudiés ${ }^{39}$,

on peut y voir l'affirmation d'un savoir-faire bien maîtrisé (l'exercice monographique) et la volonté de prouver ses capacités dans un exercice peut-être considéré comme de plus vaste envergure. C'est en effet une chance inespérée pour ces jeunes hommes d'aborder un champ d'étude presque inexploré et de pouvoir y faire des hypothèses personnelles. C'est également une manière de s'émanciper des instructions de la commission. C'est enfin un moyen de s'adapter à un terrain inconnu.

Les impressions de Dollfus et Montserrat sur l'orographie de l'Amérique centrale permettent d'analyser ce rapport à l'inconnu, la reconnaissance de l'étrangeté et la tentative de réduire cette étrangeté en la théorisant:

Au Mexique, tout est étrange et si distinct de ce que nos yeux sont accoutumés à contempler en Europe, que facilement, on se laisserait entraîner à n'y voir qu'une gigantesque ébauche à laquelle a manqué le concours des siècles... rien dans nos formations régulières, ne nous a préparés à les voir et à les comprendre, rien n'a comblé la distance qui, dans nos esprits, existe entre nos connaissances antérieures et ce qui apparaît tout à coup à nos yeux. Eh bien, ces termes de transition, c'est l'Amérique centrale qui peut nous les offrir... ${ }^{40}$.

31 Peut-être n'est-ce qu'une manière rhétorique d'évacuer la déception de ne pas avoir pu travailler au Mexique. Pourtant, cette manière d'envisager un territoire comme incompréhensible pour l'œil européen et ce besoin d'adaptation progressive se retrouvent dans la réévaluation des méthodes mêmes de travail à laquelle se livrent Dollfus et Montserrat. Dès octobre 1865, ils écrivent :

Pour faire la géologie du Mexique, on doit se résoudre à se priver d'un des moyens de travail les plus utiles en temps ordinaire, c'est l'induction. Le plus souvent en Europe, les allures générales d'une chaîne permettent de présager sa composition dans une région quelquefois fort étendue. Il n'est pas toujours absolument nécessaire d'avoir observé de près tel ou tel point pour pouvoir affirmer, presque avec certitude que certaines natures de roches doivent y apparaître ${ }^{41}$.

On se souvient des recommandations de la commission, fondées sur l'analogie et la comparaison de terrains estimés comme toujours comparables.

Cet étonnement premier, sur lequel ils reviennent plusieurs fois, oblige les géologues à la prudence: évitant une généralisation qu'ils qualifient d'absolue, ils s'astreindront d'abord

[...] à suivre pas à pas [leurs] itinéraires, reprenant une à une [leurs] observations, relevant tout ce qu'[ils ont] vu. Ce sera peut-être un peu aride mais au moins ce sera vrai $^{42}$.

Il leur faut rester dans les plus strictes limites possibles de la géologie positive où seul le fait obtenu par observation exacte se voit reconnaitre une valeur scientifique. Mais sur un terrain aussi peu connu que celui que Dollfus et Montserrat parcourent, se borner à énumérer des faits ne permet pas de donner une idée de la géologie du pays. Aussi écrivent-ils :

Après avoir accumulé des faits et avoir posé des bases solides, nous essayerons... de grouper entre elles les observations, de chercher les liaisons qui peuvent exister entre les choses et d'en déduire peut-être quelques idées plus ou moins générales $[\ldots]$

[parce qu'] aussi longtemps qu'un pays n'aura pas été étudié dans ses moindres détails, il faudra bien, pour envisager les choses d'un peu haut, laisser une certaine place à l'hypothèse, quand ce ne serait d'ailleurs que pour accumuler des idées plus 
ou moins théoriques, destinées à diriger les travaux... [ultérieurs], qu'elles doivent $y$ trouver leur confirmation ou leur destruction ${ }^{43}$.

\section{Conclusion}

34 originale d'espaces dans lesquels Dollfus et Montserrat, en se heurtant aux difficultés d'un terrain mal connu, définissent des limites, redéfinissent des savoirs et des approches. On peut déjà voir comment une modification d'itinéraire et de nouveaux lieux d'étude peuvent influer sur la pratique scientifique et comment les voyageurs proposent des solutions qui apparaissent comme les aboutissements d'un processus d'adaptation, (re)composant ainsi les contextes de leur voyage et de leur profession, un de ces multiples contextes possibles que l'on s'attachera ultérieurement à saisir dans leur diversité et leur hétérogénéité.

\section{NOTES}

1. Décret du 27 février 1864 ; arrêtés des 2 et 10 mars 1864 ; les instructions se trouvent dans les Archives de la Commission scientifique du Mexique (infra: ACSM), t. I, Paris, Imprimerie impériale, 1865.

2. Cours du 11 janvier 1996.

3. Charles Sainte-Claire-Deville (né en 1814) est membre de l'Académie des sciences. Il a participé à plusieurs expéditions scientifiques, notamment aux Antilles, au cours desquelles il se consacre surtout à l'étude des phénomène météorologiques et volcaniques.

4. Archives nationales (infra: AN), F17/2912, dossier voyageurs.

5. ACSM, t. I, rapport de V. Duruy à Napoléon III du 27 février 1864, p. 2.

6. ACSM, t. I, procès-verbal de la séance du 20 octobre 1864 .

7. ACSM, t. I, p. 37

8. Ibid., p. 40.

9. AN F17/2912, MIP, arrêté $n^{\circ} 2551$ du 9 août 1864.

10. Ami Boué, Le Guide du voyageur-géologue, Paris, 1835-1836. Ayant sillonné l'Europe, l'auteur dresse un tableau des zones à parcourir en fonction des roches à étudier. Pour les formations porphyriques et granitiques : p. 415.

11. Jean-Jacques Huot, Nouveau Manuel complet de géologie ou traité élémentaire de cette science, Paris, 1852, p. 245.

12. Adolphe d'Archiac, Histoire des progrès de la géologie, t. I, 1847, p. VIII.

13. Bernard Balan, L'Ordre et le Temps. L'anatomie comparée et l'histoire des vivants au XIXé siècle, Paris, Vrin, 1979, p. 367.

14. A. d'Archiac, op. cit., t. I et t. IV, 1851.

15. Présentation de ces travaux par Sainte-Claire-Deville aux séances des 9 mars, 22 juin 1865, 22 février 1866.

16. AN F17/2912, lettre de Dollfus à Sainte-Claire-Deville, Mexico, le 26 mars 1865.

17. ACSM, t. II, 1866, p. 132, lettre à Sainte-Claire-Deville, Mexico, le 9 mai 1865. 
18. ACSM, t. III, 1867, p. 4, Sainte-Claire-Deville, «Rapport sur divers mémoires de géologie », lettre de P. Pavie, 7 novembre 1865.

19. Ibid., lettre de Montserrat du 23 novembre 1865.

20. AN F17/2912, Dollfus à Duruy, La Union, le 3 avril 1866. Une minute du MIP datée du 8 juin accepte cette extension de parcours et accorde une indemnité supplémentaire de 1500 francs.

21. Pavie quitte alors le groupe pour retourner en France.

22. A. Dollfus, E. Montserrat, Voyage géologique dans les républiques de Guatemala et de Salvador, Mission scientifique au Mexique et dans l'Amérique centrale, Paris, 1868.

23. Ibid., p. VI.

24. Ibid., p. 178.

25. Ils utilisent également beaucoup les travaux du R. P. André Cornette, directeur de cet observatoire.

26. Par exemple E. G. Squier, The States of Central America, Their Geography, Topography, Climate..., New York, 1858 ; J. L. Stephens, Incidents of Travel in Central America, Chiapas and Yucatan, London, 1 re éd. 1841.

27. Notamment le Boletin extraordinario del gobierno del Salvador ou le Faro del Salvador.

28. ACSM, t. II, Sainte-Claire-Deville, « Rapport sur deux mémoires présentés... », p. 132.

29. A. Dollfus, E. Montserrat, op. cit., p. 342.

30. AN F17/2913, bordereau récapitulatif, ordonnancement $n^{\circ} 2060$ du 14 décembre 1864.

31. A. Dollfus, E. Montserrat, op. cit., p. 177.

32. A. Dollfus, E. Montserrat, op. cit., p. 248.

33. Par exemple : Huot, op. cit.; Boué, op. cit. . Léonce-Elie de Beaumont, Leçons de géologie pratique, professées au Collège de France pendant l'année scolaire 1843-1844, Paris, 1845.

34. A. Dollfus, E. Montserrat, op. cit., planche V.

35. Ibid., p. 248.

36. Ibid., p. 530. La carte topographique construite s'inspire des travaux de Félix Baily (1850), de Max Sonnenstern (1859), de Van den Gehühte, du père André Cornette, des cartes disséminées dans l'ouvrage de G. E. Squier, op. cit.

37. Ibid., p. IV.

38. Ibid., p. III.

39. Ibid., p. V.

40. Ibid., p. 85. On voit ici comment l'appréhension du terrain va de pair avec une approche européocentrée du temps géologique.

41. ACSM, t. II, Mémoires et notes géologiques, p. 364-403.

42. A. Dollfus, E. Montserrat, op. cit., p. 178.

43. Ibid., p. 247.

\section{AUTEUR}

\section{CLAIRE FRED}

Diplômée de l'Institut d'études politiques (IEP), Paris, 1992.

EHESS, sous la direction de Bernard Lepetit :

DEA « Histoire et civilisations », 1993. 
Doctorante.

Professeur agrégée d'histoire. 